\title{
A REPRESENTATION THEOREM AND APPROXIMATION OPERATORS ARISING FROM INEQUALITIES INVOLVING DIFFERENTIAL OPERATORS
}

\author{
BY \\ D. LEVIATAN( $\left.{ }^{(}\right)$
}

\begin{abstract}
A representation of functions as integrals of a kernel $\psi(t ; x)$, which was introduced by Studden, with respect to functions of bounded variation in $[0, \infty)$ is obtained whenever the functions satisfy some conditions involving the differential operators $(d / d t)\left\{f(t) / w_{i}(t)\right\}, i=0,1,2, \ldots$. The results are related to the concepts of generalized completely monotonic functions and generalized absolutely monotonic functions in $(0, \infty)$. Some approximation operators for the approximation of continuous functions in $[0, \infty)$ arise naturally and are introduced; some sequenceto-function summability methods are also introduced.
\end{abstract}

1. Introduction and statement of main results. Let $\left\{w_{i}(t)\right\}_{i=0}^{\infty}$ be an infinite sequence of functions which are positive on $[0, \infty)$ and each belongs to $C^{\infty}[0, \infty)$. Define a sequence of differential operators, $D_{i}$, by

$$
D_{i} f(t)=\frac{d}{d t} \frac{f(t)}{w_{i}(t)}, \quad i=0,1,2, \ldots
$$

In a recent article Studden [7] has discussed in detail the solutions of the following system of infinitely many differential inequalities

$$
\begin{aligned}
\phi(t) \in C^{\infty}(0, \infty), \quad \phi(t) \geqq 0 \quad \text { and } \quad & (-1)^{k} D_{k-1} D_{k-2} \cdots D_{0} \phi(t) \geqq 0 \\
& \text { for all } 0<t<\infty \text { and } k=1,2, \ldots .
\end{aligned}
$$

A solution of (1.2) is called a generalized completely monotonic (abbreviated GCM) function on $(0, \infty)$ with respect to $\left\{w_{i}(t)\right\}$. Assuming that there exists a function $f(t)$ on $[0, \infty)$ such that

$$
0<f(t)<1, \quad t \in[0, \infty) ; \quad \lim _{t \rightarrow \infty} f(t)=0 ;
$$

$$
\int_{0}^{\infty} f(t) d t<\infty ; \quad \inf (1-f(t))>0
$$

$$
1-f(t) \leqq w_{i}(t) \leqq 1+f(t), \quad t \in[0, \infty), \quad i=1,2, \ldots
$$

Received by the editors August 31, 1970 and, in revised form, March 1, 1971.

AMS 1969 subject classifications. Primary 4430, 4130; Secondary 3490, 4031.

Key words and phrases. Generalized completely monotonic functions, representation of functions as integrals of a kernel, approximation operators in $[0, \infty)$, sequence-to-function summability methods.

( $\left.{ }^{1}\right)$ Research partially supported by NSF grant GP-14592 to the University of Illinois.

Copyright (C) 1972, American Mathematical Society 
and assuming further that $w_{0}(t) \equiv 1$ (otherwise $\phi$ is replaced by $\phi / w_{0}$ ) Studden has represented the GCM functions as integrals of a kernel $\psi(t ; x)$, which is defined by means of $\left\{w_{i}(t)\right\}$ only, with respect to a Borel measure $\mu$, on $[0, \infty)$.

The closely related topic of generalized absolutely monotonic (GAM) functions on a finite interval $(a, b)$ was discussed by Karlin and Ziegler [5] (see also Karlin and Studden [4]). They, under some mild conditions on $\left\{w_{i}(t)\right\}$, expanded each GAM function on $(a, b)$ into a "power"-series.

Let $w_{0}(t) \equiv 1, t \in[0, \infty)$. Set, for $n \geqq 1$,

$$
\begin{aligned}
\psi_{n}(t ; x) & =\int_{t}^{x} w_{n}\left(\xi_{n}\right) \int_{t}^{\xi_{n}} w_{n-1}\left(\xi_{n-1}\right) \cdots \int_{t}^{\xi_{2}} w_{1}\left(\xi_{1}\right) d \xi_{1} \cdots d \xi_{n}, & & 0 \leqq t \leqq x, \\
& =0, & & t>x,
\end{aligned}
$$

and set $\psi_{0}(t ; x)=1,0 \leqq t \leqq x ; \psi_{0}(t ; x)=0, t>x$. Now denote

$$
\begin{aligned}
G_{k} \phi(u) & =(-1)^{k} D_{k-1} D_{k-2} \cdots D_{0} \phi(u), \quad k=1,2, \ldots, \\
G_{0} \phi(u) & =\phi(u) .
\end{aligned}
$$

Given a function $\phi \in C^{n+1}(0, \infty)$ it follows by integration by parts (see [7, (4.12)]) that for $t, u, 0<t<u<\infty$,

$$
\phi(t)=\sum_{k=0}^{n} \psi_{k}(t ; u) \frac{G_{k} \phi(u)}{w_{k}(u)}+\int_{t}^{u} \psi_{n}(t ; x) G_{n+1} \phi(x) d x .
$$

This is a sort of "finite Taylor expansion" of $\phi$ by means of the "derivatives" $G_{k} \phi$. The natural question arises as to under what conditions is it possible to expand a function $\phi \in C^{\infty}(0, \infty)$ into the "Taylor series"

$$
\phi(t)=\sum_{k=0}^{\infty} \psi_{k}(t ; u) \frac{G_{k} \phi(u)}{w_{k}(u)}, \quad u \geqq t>0 .
$$

In case $\phi$ is a GCM function, an adaption of some work of Karlin and Ziegler [5] yields mild conditions under which any GCM function can be expanded into the series (1.7). We shall omit the details here as the results will appear as consequences of ours.

Evidently, in case $\phi$ is a GCM function, the series on the right-hand side of (1.7) converges absolutely for each pair $t>0, u>0$ (if $u<t$, then the series is composed of zeros). Furthermore, for a fixed $t>0$, the series is bounded (by $\phi(t)$ ) uniformly in $u>0$. If $\phi(0+)<\infty$, then the series is bounded (by $\phi(0+))$ uniformly in $t \geqq 0$ and $u>0$. Therefore one arrives at the question of characterizing those functions $\phi \in C^{\infty}(0, \infty)$ for which the series on the right-hand side of (1.7) converges absolutely, or converges to $\phi(t)$, or both. This apparently is a difficult problem and we are only able to give a partial answer.

Of the following three theorems we shall prove the first and leave the proof of the other two to the reader. (They are derived from the proof of the first theorem in much the same way that results in [5] were derived.) 
THEOREM 1. Let $m_{i}(c ; d)$ and $M_{i}(c ; d)$ be defined by

$$
m_{i}(d ; c)=\min _{d \leqq t \leqq c} w_{i}(t) \leqq \max _{d \leqq t \leqq c} w_{i}(t)=M_{i}(d ; c), \quad i=1,2, \ldots
$$

Suppose that for every $c \in(0, \infty)$ there exist $a d, 0<d<c$, and an $\varepsilon>0$ such that

$$
\lim _{n \rightarrow \infty}\left(\prod_{i=1}^{n} \frac{M_{i}(c ; d)}{m_{i}(c ; d)}\right) \varepsilon^{n}=0
$$

Then if, for some $\tau \geqq 0, \phi \in C^{\infty}(\tau, \infty)$ and, for some $t_{0} \geqq 0$, the series

$$
\sum_{k=0}^{\infty} \psi_{k}\left(t_{0} ; u\right) \frac{\left|G_{k} \phi(u)\right|}{w_{k}(u)}
$$

is bounded uniformly in $u$ in compact subsets of $(\tau, \infty)$, then $\phi(t)$ admits the expansion (1.7) for all $u, t, u \geqq t>\max \left(\tau, t_{0}\right)$.

Remark 1. Condition (1.8) is fulfilled for instance if the functions $w_{i}(t)$ are uniformly bounded above and below. This is the case if (1.3) is satisfied.

THEOREM 2. If $w_{i}(t), i=1,2, \ldots$, are nonincreasing in $[0, \infty]$ and if the assumptions on $\phi$ of Theorem 1 are fulfilled, then the conclusion of Theorem 1 is valid.

THEOREM 3. Suppose there exist two sequences $\left\{c_{n}\right\},\left\{d_{n}\right\}$ such that, for all $n$ sufficiently large,

$$
c_{n}(x-t)^{n} / n ! \leqq \psi_{n}(t ; x) \leqq d_{n}(x-t)^{n} / n !, \quad x \geqq t,
$$

and such that, for some $\varepsilon>0$,

$$
\lim _{n \rightarrow \infty} \frac{d_{n}}{c_{n}} \varepsilon^{n}=0 .
$$

Then if the assumptions on $\phi$ of Theorem 1 are fulfilled, then the conclusion of Theorem 1 is valid.

The following is now an immediate consequence of the above discussion and theorems.

COROLLARY 1. Under the assumptions on $\left\{w_{i}(t)\right\}$ in either one of the preceding theorems, each GCM function on $(0, \infty)$ possesses the expansion (1.7).

Another consequence whose proof will be given after the proof of Theorem 1 is the following.

COROLlaRY 2. Suppose $\left\{w_{i}(t)\right\}$ satisfies the assumptions of one of the above theorems and that $\phi \in C^{\infty}(\tau, \infty)$ for some $\tau \geqq 0$. Then the series (1.9) is bounded uniformly in $u$ in compact subsets of $(\tau, \infty)$, for some $t_{0} \geqq 0$, if and only if $\phi$ is the difference of two GCM functions in the interval $\left(\max \left(\tau, t_{0}\right), u\right)$ for every $u>\max \left(\tau, t_{0}\right)$. 
Under (1.3) we shall give a complete characterization of the case where the series (1.9) is bounded uniformly in $u>0$. This characterization turns out to be even more interesting due to its nature.

TheOREM 4. Let $\left\{w_{i}(t)\right\}$ satisfy (1.3) and let $\phi \in C^{\infty}(0, \infty)$. Then a necessary and sufficient condition in order that

$$
\sup _{u>0} \sum_{k=0}^{\infty} \psi_{k}(0 ; u) \frac{\left|G_{k} \phi(u)\right|}{w_{k}(u)}=H<\infty
$$

is that there exists an $\alpha(x)$ of bounded variation in $[0, \infty)$ such that

$$
\int_{0}^{\infty}|d \alpha(x)|=H
$$

and

$$
\phi(t)=\int_{0}^{\infty} \psi(t ; x) d \alpha(x), \quad t>0,
$$

where $\psi(t ; x)$ is the kernel of Studden [7, (2.7)].

RemarK 2. (a) Condition (1.12) corresponds to $\phi(0+)<\infty$ in the case of the GCM functions.

(b) The proof of Theorem 4 provides us with a different proof of [7, Theorem 1]. Studden's proof, however, seems to work only for $t>2 F(\infty)$.

(c) By virtue of Theorem 1, if (1.12) is fulfilled, then $\phi(t)$ admits the expansion (1.7) for all $u, t, u \geqq t>0$.

Our following result is of the nature of Theorem 1 but involves an entirely different condition on $\left\{w_{i}(t)\right\}$. As it turns out, the proof of this theorem is much shorter than that of Theorem 1.

THEOREM 5. Let $\phi \in C^{\infty}(\tau, \infty)$ for some $\tau \geqq 0$ and assume that for some $t_{1} \geqq 0$

$$
\sum_{k=0}^{\infty} \min _{t \leqq x \leqq u}\left\{\frac{\psi_{k+1}\left(t_{1} ; x\right)}{w_{k+1}(x) \psi_{k}\left(t_{1} ; x\right)}\right\}=\infty \quad \text { for all } u, t\left(u \geqq t>t_{1}\right) .
$$

Then if for some $t_{0}, 0 \leqq t_{0} \leqq t_{1}$, the series (1.9) is bounded uniformly in $u$ in compact subsets of $(\tau, \infty)$, then $\phi(t)$ admits the expansion (1.7) for all $u, t, u \geqq t \geqq \max \left(\tau, t_{1}\right)$.

Remark 3. Condition (1.15) is satisfied for example when (1.10) holds for all sufficiently large $n$ and the sequences $\left(c_{n}\right),\left(d_{n}\right)$ satisfy

$$
\sum_{n=0}^{\infty} \frac{c_{n} c_{n+1}}{d_{n} d_{n+1}} \frac{1}{n+1}=\infty
$$

since $\max _{t \leqq x \leqq u} w_{n+1}(x) \leqq d_{n+1} / c_{n}$.

The last result to be mentioned in this section deals only with GCM functions. We were unable to extend it to the more general case. The proof here is an adaptation of the technique of Amir and Ziegler [1] and is left to the reader. 
THEOREM 6. A necessary and sufficient condition that each GCM function on $(0, \infty)$ can be expanded into the series $(1.7)$ is that for each $u$ and $t(u>t)$ there exists an $s(0<s<t)$ for which

$$
\lim _{n \rightarrow \infty} \frac{\psi_{n}(t ; u)}{\psi_{n}(s ; u)}=0
$$

In $\S 3$ we shall introduce some approximation operators which arise naturally from our discussion and which generalize the Szász operators [8], while in $\S 4$ we shall define by means of the $G_{k} \phi$ 's, some sequence-to-function summability methods which generalize the $[J, f(x)]$ transforms [3].

\section{Proofs.}

Proof of Theorem 1. Let $\phi \in C^{\infty}(\tau, \infty)$ be such that (1.9) holds and fix $u, t$, $u \geqq t>\max \left(\tau, t_{0}\right)$. Since $\psi_{k}(d ; x) \leqq \psi_{k}\left(t_{0} ; x\right)$ for $t \leqq d \leqq x \leqq u$ and since $[t, u]$ is compact it follows by (1.9) that

$$
\sum_{k=0}^{\infty} \psi_{k}(d ; x) \frac{\left|G_{k} \phi(x)\right|}{w_{k}(x)} \leqq H, \quad t \leqq d \leqq x \leqq u .
$$

By virtue of (1.6) our proof will be complete once we show that

$$
\lim _{n \rightarrow \infty} \int_{t}^{u} \psi_{n}(t ; x) G_{n+1} \phi(x) d x=0 .
$$

Now by (2.1) the series on the right-hand side of (1.6) converges as $n \rightarrow \infty$, hence define

$$
\begin{aligned}
g(d) & =\phi(d)-\sum_{k=0}^{\infty} \psi_{k}(d ; u) \frac{G_{k} \phi(u)}{w_{k}(u)} \\
& =\lim _{n \rightarrow \infty} \int_{d}^{u} \psi_{n}(d ; x) G_{n+1} \phi(x) d x .
\end{aligned}
$$

We have to show that $g(t)=0$. We shall show, following the technique of Karlin and Ziegler [5], that $g(d)=0, t \leqq d \leqq u$.

Our proof is done in steps. First by (2.1):

$$
\left|G_{n} \phi(x)\right| \leqq H \frac{w_{n}(x)}{\psi_{n}(d ; x)}, \quad t \leqq d<x \leqq u, \quad n=0,1,2, \ldots
$$

We shall prove that whenever a function $\phi \in C^{\infty}(t, u]$ satisfies (2.4), then for every $c, t<c \leqq u$, there is an $\varepsilon>0$ such that if $c-\varepsilon \leqq b \leqq c$ then

$$
\lim _{n \rightarrow \infty} \int_{b}^{c} \psi_{n}(b ; x) G_{n+1} \phi(x) d x=0
$$


Then we shall prove that $g \in C^{\infty}(t, u]$, that it satisfies (2.4) and that $g(d) \equiv 0$, $t \leqq d \leqq u$. Now by (2.4), for $t \leqq d<b<c$,

$$
\begin{aligned}
\int_{b}^{c} \psi_{n}(b ; x)\left|G_{n+1} \phi(x)\right| d x & \leqq H \int_{b}^{c} \psi_{n}(b ; x) \frac{w_{n+1}(x)}{\psi_{n+1}(d ; x)} d x \\
& \leqq H \frac{\psi_{n+1}(b ; c)}{\psi_{n+1}(d ; b)} \\
& \leqq H\left(\prod_{i=1}^{n+1} \frac{M_{i}(d ; c)}{m_{i}(d ; c)}\right)\left(\frac{c-b}{b-d}\right)^{n+1} \rightarrow 0 \text { as } n \rightarrow \infty,
\end{aligned}
$$

by virtue of (1.8), provided $\varepsilon$ is properly chosen. This proves (2.5).

We now prove that $g \in C^{\infty}(t, u]$ and at the same time produce $G_{k} g(d)$. An easy computation shows that

$$
\begin{aligned}
G_{k} \psi_{n}(d ; u) & =w_{k}(d) \psi_{n-k}(d ; u ; k), & & n \geqq k, \\
& =0, & & n<k,
\end{aligned}
$$

where, for $n>k$ and $d \leqq u$,

$$
\psi_{n-k}(d ; u ; k)=\int_{d}^{u} w_{n}\left(\xi_{n}\right) \int_{d}^{\xi_{n}} w_{n-1}\left(\xi_{n-1}\right) \cdots \int_{d}^{\xi_{k+2}} w_{k+1}\left(\xi_{k+1}\right) d \xi_{k+1} \cdots d \xi_{n},
$$

and $\psi_{0}(d ; u ; k)=1$. Hence, formal differentiation of (2.3) yields

$$
G_{k} g(d)=G_{k} \phi(d)-\sum_{n=k}^{\infty} w_{k}(d) \psi_{n-k}(d ; u ; k) \frac{G_{n} \phi(u)}{w_{n}(u)}, \quad t<d \leqq u .
$$

To justify the term-by-term differentiation we observe that, for $t \leqq t_{1}<d \leqq u$,

$$
\begin{aligned}
& \psi_{k}\left(t_{1} ; d\right) \psi_{n-k}(d ; u ; k) \\
& \quad=\int_{t_{1}}^{d} w_{k}\left(\xi_{k}\right) \cdots \int_{t_{1}}^{\xi_{2}} w_{1}\left(\xi_{1}\right) d \xi_{1} \cdots d \xi_{k} \cdot \int_{d}^{u} w_{n}\left(\xi_{n}\right) \cdots \int_{d}^{\xi_{k+2}} w_{k+1}\left(\xi_{k+1}\right) d \xi_{k+1} \cdots d \xi_{n} \\
& \quad=\int_{d}^{u} w_{n}\left(\xi_{n}\right) \cdots \int_{d}^{\xi_{k+2}} w_{k+1}\left(\xi_{k+1}\right) \int_{t_{1}}^{d} w_{k}\left(\xi_{k}\right) \cdots \int_{t_{1}}^{\xi_{2}} w_{1}\left(\xi_{1}\right) d \xi_{1} \cdots d \xi_{n} \\
& \quad \leqq \int_{t_{1}}^{u} w_{n}\left(\xi_{n}\right) \cdots \int_{t_{1}}^{\xi_{2}} w_{1}\left(\xi_{1}\right) d \xi_{1} \cdots d \xi_{n}=\psi_{n}\left(t_{1} ; u\right) .
\end{aligned}
$$

Whence

$$
\sum_{n=k}^{\infty} w_{k}(d) \psi_{n-k}(d ; u ; k) \frac{\left|G_{n} \phi(u)\right|}{w_{n}(u)} \leqq \frac{w_{k}(d)}{\psi_{k}\left(t_{1} ; d\right)} \sum_{n=k}^{\infty} \psi_{n}\left(t_{1} ; u\right) \frac{\left|G_{n} \phi(u)\right|}{w_{n}(u)}
$$

where, by (2.1), the series on the right-hand side converges, and it follows from the nature of the dependence on $d$ on the right-hand side that the series on the lefthand side converges uniformly in $d$ in compact subsets of $\left(t_{1}, u\right]$. This justifies (2.6). 
Appealing to (2.7) once again, it follows by (2.1), (2.4) and (2.6) that

$$
\left|G_{n} g(d)\right| \leqq 2 H w_{n}(d) / \psi_{n}\left(t_{1} ; d\right), \quad t \leqq t_{1}<d \leqq u .
$$

Now (2.5) for $c=u$ shows us that $g(d)=0$ in some proper interval $[u-\varepsilon, u]$. Let $c_{0}=\inf \{c \mid g(d)=0$ for all $d \in[c, u]\}$ and suppose $c_{0}>t$. Then (1.6) with $g$ replacing $\phi$ gives

$$
g(d)=\int_{d}^{c_{0}} \psi_{n}(d ; x) G_{n+1} g(x) d x
$$

(since $G_{n} g(x)=0$ for $c_{0}<x \leqq u, n=0,1,2, \ldots$ ). Consequently,

$$
g(d)=\lim _{n \rightarrow \infty} \int_{d}^{c_{0}} \psi_{n}(d ; x) G_{n+1} g(x) d x,
$$

but by virtue of (2.8) and the proof of (2.5), there exists an $\varepsilon_{1}>0$ such that

$$
g(d)=\lim _{n \rightarrow \infty} \int_{d}^{c_{0}} \psi_{n}(d ; x) G_{n+1} g(x) d x=0, \quad d \in\left[c_{0}-\varepsilon_{1}, c_{0}\right],
$$

contradicting the definition of $c_{0}$. Therefore $c_{0}=t$ and, as $g(d)$ evidently is continuous in $[t, u], g(d) \equiv 0, d \in[t, u]$, and the proof is complete.

Proof of Corollary 2. Sufficiency is obvious by the discussion preceding Theorem 1 , so we prove only necessity. Let $u>\max \left(\tau, t_{0}\right)$. By either one of Theorems 1,2 or $3, \phi$ satisfies (1.7) for all $u \geqq t>\max \left(\tau, t_{0}\right)$. Hence, for $u \geqq t$ $>\max \left(\tau, t_{0}\right)$,

$$
\begin{aligned}
\phi(t) & =\sum_{k=0}^{\infty} \psi_{k}(t ; u) \frac{\left|G_{k} \phi(u)\right|+G_{k} \phi(u)}{2 w_{k}(u)}-\sum_{k=0}^{\infty} \psi_{k}(t ; u) \frac{\left|G_{k} \phi(u)\right|-G_{k} \phi(u)}{2 w_{k}(u)} \\
& =\phi_{1}(t)-\phi_{2}(t), \text { say. }
\end{aligned}
$$

Now by the proof of (2.6) it follows that each of $\phi_{i}$ is a GCM function in $\left(\max \left(\tau, t_{0}\right), u\right)$ and this completes the proof.

We shall defer the proof of Theorem 4 until after we prove Theorem 5 due to the shortness of the proof of the latter.

Proof of Theorem 5. Here again (1.6) is valid so that we are done once we establish (2.2). Fix $u, t, u \geqq t>\max \left(\tau, t_{1}\right)$. By (1.9),

Therefore

$$
\sum_{k=0}^{\infty} \psi_{k}\left(t_{1} ; x\right) \frac{\left|G_{k} \phi(x)\right|}{w_{k}(x)} \leqq H, \quad t \leqq x \leqq u .
$$

$$
\begin{aligned}
\infty & >H(u-t) \geqq \sum_{k=0}^{\infty} \int_{t}^{u} \psi_{k+1}\left(t_{1} ; x\right) \frac{\left|G_{k+1} \phi(x)\right|}{w_{k+1}(x)} d x \\
& =\sum_{k=0}^{\infty} \int_{t}^{u} \frac{\psi_{k+1}\left(t_{1} ; x\right)}{w_{k+1}(x) \psi_{k}\left(t_{1} ; x\right)} \psi_{k}\left(t_{1} ; x\right)\left|G_{k+1} \phi(x)\right| d x \\
& \geqq \sum_{k=0}^{\infty} \min _{t \leqq x \leqq u}\left\{\frac{\psi_{k+1}\left(t_{1} ; x\right)}{w_{k+1}(x) \psi_{k}\left(t_{1} ; x\right)}\right\} \int_{t}^{u} \psi_{k}\left(t_{1} ; x\right) G_{k+1} \phi(x) d x .
\end{aligned}
$$


By virtue of (1.15), this implies that for a subsequence $k_{i} \rightarrow \infty$

$$
\lim _{i \rightarrow \infty} \int_{t}^{u} \psi_{k_{i}}\left(t_{1} ; x\right)\left|G_{k_{\imath}+1} \phi(x)\right| d x=0
$$

which in turn implies

$$
\lim _{i \rightarrow \infty} \int_{t}^{u} \psi_{k_{i}}(t ; x)\left|G_{k_{i}+1} \phi(x)\right| d x=0 .
$$

Now (1.9) guarantees the existence of the limit on the right-hand side of (2.3) and therefore (2.9) implies (2.2). This completes the proof.

Proof of Theorem 4. First we prove sufficiency. We show first that

$$
\sum_{k=0}^{\infty} \psi_{k}(0 ; t) \frac{G_{k} \psi(t ; x)}{w_{k}(t)} \leqq 1 \text { for all } x, t \in[0, \infty)
$$

To this end recall (1.6) for the function $\psi(t ; x)$ which as a function of $t$ is a GCM function in $[0, \infty)$. For every $n \geqq 0$ and $x, t \in[0, \infty)$ we have

$$
1=\psi(0 ; x)=\sum_{k=0}^{n} \psi_{k}(0 ; t) \frac{G_{k} \psi(t ; x)}{w_{k}(t)}+\int_{0}^{t} \psi_{n}(0 ; u) G_{n+1} \psi(u ; x) d u .
$$

Hence

$$
1 \geqq \sum_{k=0}^{n} \psi_{k}(0 ; t) \frac{G_{k} \psi(t ; x)}{w_{k}(t)}, \quad n=0,1,2, \ldots, \quad x, t \in[0, \infty),
$$

which establishes (2.10). Now (2.10), together with [7, (3.3)], implies that $0 \leqq G_{k} \psi(t ; x) \leqq w_{k}(t) / \psi_{k}(0 ; t), t>0$, therefore we are allowed to differentiate under the sign of the integral in (1.14) and thus have

$$
G_{k} \phi(t)=\int_{0}^{\infty} G_{k} \psi(t ; x) d \alpha(x), \quad t>0
$$

Hence by (2.10)

$$
\begin{aligned}
\sum_{k=0}^{\infty} \psi_{k}(0 ; t) \frac{\left|G_{k} \phi(t)\right|}{w_{k}(t)} & \leqq \int_{0}^{\infty} \sum_{k=0}^{\infty} \psi_{k}(0 ; t) \frac{G_{k} \psi(t ; x)}{w_{k}(t)}|d \alpha(x)| \\
& \leqq \int_{0}^{\infty}|d \alpha(x)|<\infty
\end{aligned}
$$

This proves sufficiency and shows that

$$
H \leqq \int_{0}^{\infty}|d \alpha(x)| .
$$

Conversely, as we have remarked in Remark 2(c), by Theorem 1, (1.11) implies that

$$
\phi(t)=\sum_{k=0}^{\infty} \psi_{k}(t ; u) \frac{G_{k} \phi(u)}{w_{k}(u)}, \quad u \geqq t>0 .
$$


Rewriting this yields

$$
\begin{aligned}
\phi(t) & =\sum_{k=0}^{\infty} \frac{\psi_{k}(t ; u)}{\psi_{k}(0 ; u)} \psi_{k}(0 ; u) \frac{G_{k} \phi(u)}{w_{k}(u)} \\
& =\sum_{k=0}^{\infty} \frac{\psi_{k}\left(t ; k / u_{k}\right)}{\psi_{k}\left(0 ; k / u_{k}\right)} \psi_{k}(0 ; u) \frac{G_{k} \phi(u)}{w_{k}(u)}, \quad u \geqq t>0,
\end{aligned}
$$

where $u_{k}=k / u$ (for $k=0$ we understand $k / u_{k}$ as $u$ ). Now [7, (2.7) and (2.8)] $\psi(t ; x)$ $=e^{-x t} P(t ; x) / P(0 ; x), x, t \in[0, \infty)$, where

$$
P(t ; x)=1+\int_{t}^{\infty} e^{-x(\xi-t)} \sum_{k=0}^{\infty} x^{k}\left(w_{k}(\xi)-1\right) \psi_{k-1}(t ; \xi) d \xi
$$

Denote $F(t)=\int_{0}^{t} f(\tau) d \tau$ where $f$ is the function of (1.3). Then, by (1.3), $0<F(\infty)$ $<\infty$, and by $[7,(3.2)]$ it follows that

$$
0 \leqq \psi(t ; x) \leqq e^{-x(t-2 F(\infty))} .
$$

Consequently, for any fixed $t>2 F(\infty), \psi(t ; x) \rightarrow 0$ as $x \rightarrow \infty$. We are now able to apply [7, Lemma 4] and conclude that the functions

$$
\begin{aligned}
\Phi_{n}(t ; x) & =\psi_{n}(t ; n / x) / \psi_{n}(0 ; n / x), & & n \geqq t x, x \neq 0, \\
& =1, & & x=0, \\
& =0, & & n<t x,
\end{aligned}
$$

converge to $\psi(t ; x)$ uniformly in $0 \leqq x<\infty$, for every fixed $t>2 F(\infty)$. Also, for each fixed $k, \psi_{k}(t ; u) / \psi_{k}(0 ; u) \rightarrow 1$, as $u \rightarrow \infty$, and $\psi(t, k / u) \rightarrow \psi(t ; 0+)=1$, as $u \rightarrow \infty$. Therefore by (1.12) and (2.14) we have

$$
\phi(t)=\lim _{u \rightarrow \infty} \sum_{k=0}^{\infty} \psi(t ; k / u) \psi_{k}(0 ; u) \frac{G_{k} \phi(u)}{w_{k}(u)}, \quad t>2 F(\infty) .
$$

If we define the functions $\alpha_{u}(x)$ by

$$
\begin{aligned}
& \alpha_{u}(0)=0, \\
& \alpha_{u}(x)=\sum_{k \leqq u x} \psi_{k}(0 ; u) \frac{G_{k} \phi(u)}{w_{k}(u)}, \quad 0<x<\infty,
\end{aligned}
$$

then the functions $\alpha_{u}(x)$ are of uniformly bounded variations in $[0, \infty)$ and $(2.16)$ takes the form

$$
\phi(t)=\int_{0}^{\infty} \psi(t ; x) d \alpha_{u}(x), \quad t>2 F(\infty)
$$

Now since $\psi(t ; \infty)=0$ for $t>2 F(\infty)$ it follows by [2, Theorem 5.1] that there exists an $\alpha(x)$ of variation not exceeding $H$ such that

$$
\phi(t)=\int_{0}^{\infty} \psi(t ; x) d \alpha(x), \quad t>2 F(\infty)
$$


But $0 \leqq \psi(t ; x) \leqq 1$ for all $t, x \in[0, \infty)$, hence the integral in (2.18) exists for all $t>0$; so define $\chi(t)$ by

$$
\chi(t)=\int_{0}^{\infty} \psi(t ; x) d \alpha(x), \quad t>0
$$

Evidently $G_{k}(\phi-\chi)(u)=0$ for $u>2 F(\infty)$ and by the sufficiency part and Theorem 1, it follows that, for $0<t \leqq 2 F(\infty)<u$,

$$
\phi(t)-\chi(t)=\sum_{k=0}^{\infty} \psi_{k}(t ; u) \frac{G_{k}(\phi-\chi)(u)}{w_{k}(u)}=0 .
$$

Therefore,

$$
\phi(t)=\int_{0}^{\infty} \psi(t ; x) d \alpha(x), \quad t>0
$$

and the proof of (1.14) is complete. Also it was noted that $\int_{0}^{\infty}|d \alpha(x)| \leqq H$ so, together with (2.13), (1.13) is established.

In fact, if $\alpha(x)$ is chosen to be normalized, i.e. $\alpha(x)=\frac{1}{2}[\alpha(x+)+\alpha(x-)], 0<x<\infty$, then by the uniqueness of $\alpha(x)$ [7, Theorem 2] we have $\lim _{u \rightarrow \infty} \alpha_{u}(t)=\alpha(t)$ at every point of continuity, $t$, of $\alpha(x)$.

3. The approximation operators. Throughout this section we assume that $\left\{w_{i}(t)\right\}$ satisfies (1.3).

Let $u>0$ be arbitrary. Then the function $\psi(t ; u)$ is represented by

$$
\psi(t ; u)=\int_{0}^{\infty} \psi(t ; x) d \alpha_{u}(x), \quad t>0,
$$

where $\alpha_{u}(x)=0,0 \leqq x<u ; \alpha_{u}(x)=1, u \leqq x<\infty$. Our discussion in the proof of Theorem 4 yields

$$
\begin{aligned}
\lim _{v \rightarrow \infty} \sum_{k / v \leqq x} \psi_{k}(0 ; v) \frac{G_{k} \psi(v ; u)}{w_{k}(v)} & =0, & & 0<x<u, \\
& =1, & & u<x<\infty,
\end{aligned}
$$

or rewriting it, we have for $u, x>0, u \neq x$,

$$
\lim _{v \rightarrow \infty} \sum_{k=0}^{\infty} \psi_{k}(0 ; v) \frac{G_{k} \psi(v ; u)}{w_{k}(v)}\left[1-\alpha_{x}\left(\frac{k}{v}\right)\right]=1-\alpha_{x}(u)
$$

This suggests the introduction of the following approximation operators. With a function $f(t)$ which is defined in $[0, \infty)$ associate the family of functions $S_{u} f$ defined on $[0, \infty)$ by

$$
S_{u} f(x)=\sum_{k=0}^{\infty} \psi_{k}(0 ; u) \frac{G_{k} \psi(u ; x)}{w_{k}(u)} f\left(\frac{k}{u}\right), \quad u>0
$$


provided the series on the right-hand side of (3.2) converges. By virtue of (2.10) the operators are well defined for every function $f(t)$ which is bounded in $[0, \infty)$. In fact, it is not difficult to prove with the help of (3.1) that if $f(t)$ is bounded in $[0, \infty)$ and continuous at $0 \leqq x<\infty$, then

$$
\lim _{u \rightarrow \infty} S_{u} f(x)=f(x)
$$

We shall prove much more.

THEOREM 7. Let $f(t)$ be defined in $[0, \infty)$ and assume that $f(t)=O\left(e^{A t}\right)$ for some $A \geqq 0$. Then (i) (3.3) holds at every point of continuity, $x$, of $f(t)$; (ii) if $f(t)$ is continuous in $[a, b]$ then (3.3) holds uniformly in $x \in[a, b]$.

RemarK 4. (a) In Theorem 7 we implicitly state that whenever $f(t)=O\left(e^{A t}\right)$, then $S_{u} f$ is well defined for all $u>0$.

(b) In the case $w_{i}(t) \equiv 1, i=0,1,2, \ldots$, the operators $S_{u}$ reduce to the wellknown Szász operators [8]

$$
S_{u} f(x)=e^{-x u} \sum_{k=0}^{\infty} \frac{(u x)^{k}}{k !} f\left(\frac{k}{u}\right)
$$

and Theorem 7 in this case is well known.

Proof of Theorem 7. First we show that whenever $f(t)=O\left(e^{A t}\right)$ for some $A \geqq 0$, then $S_{u} f$ is well defined. By [7, (3.1) through (3.4)]

$$
0 \leqq \psi_{k}(0 ; u) \leqq \frac{(u+F(u))^{k}}{k !}
$$

and

$$
0 \leqq G_{k} \psi(u ; x) \leqq x^{k} w_{k}(u) e^{-x(u-2 F(\infty))}
$$

Hence

$$
\begin{aligned}
\sum_{k=0}^{\infty} \psi_{k}(0 ; u) \frac{G_{k} \psi(u ; x)}{w_{k}(u)}\left|f\left(\frac{k}{u}\right)\right| & \leqq e^{-x(u-2 F(\infty))} \sum_{k=0}^{\infty} \frac{(u+F(u))^{k}}{k !} x^{k}\left(e^{A / u}\right)^{k} \\
& =\exp \left\{-x(u-2 F(\infty))+x e^{A / u}(u+F(u))\right\}<\infty
\end{aligned}
$$

Now we observe that by (2.11) it follows from the proof of Theorem 1 that

$$
\sum_{k=0}^{\infty} \psi_{k}(0 ; u) \frac{G_{k} \psi(u ; x)}{w_{k}(u)}=1, \quad u, x \in[0, \infty),
$$

so that we get

$$
S_{u} f(x)-f(x)=\sum_{k=0}^{\infty} \psi_{k}(0 ; u) \frac{G_{k} \psi(u ; x)}{w_{k}(u)}\left[f\left(\frac{k}{u}\right)-f(x)\right] .
$$

Let $x \in[0, \infty)$ be a point of continuity of $f(t)$ and let $\varepsilon>0$ be prescribed. Then 
there exist $u_{0}>0$ and $\delta>0$ such that if $u \geqq u_{0}$ and $\left|k-x(u+F(u)) e^{A / u}\right|<\delta u$, then $|f(k / u)-f(x)|<\varepsilon$. By (3.6), for $u \geqq u_{0}$, we then have

$$
\left|S_{u} f(x)-f(x)\right| \leqq\left\{\sum_{1}+\sum_{2}\right\} \psi_{k}(0 ; u) \frac{G_{k} \psi(u ; x)}{w_{k}(u)}\left|f\left(\frac{k}{u}\right)-f(x)\right|,
$$

where $\sum_{1}$ is taken on all $k,\left|k-x(u+F(u)) e^{A / u}\right|<\delta u$ and $\sum_{2}$ is taken on the rest. It follows by (3.5) and (3.6) that

$$
\sum_{1} \psi_{k}(0 ; u) \frac{G_{k} \psi(u ; x)}{w_{k}(u)}\left|f\left(\frac{k}{u}\right)-f(x)\right|<\varepsilon .
$$

Set $y=x(u+F(u)) e^{A / u}$. Then

$$
\begin{aligned}
\sum_{2} \psi_{k}(0 ; u) \frac{G_{k} \psi(u ; x)}{w_{k}(u)}\left|f\left(\frac{k}{u}\right)-f(x)\right| \\
\leqq e^{-x(u-2 F(\infty))}\left(1+e^{A x}\right) \sum_{2} \frac{y^{k}}{k !} \\
\leqq C(x) e^{-x u} \delta^{-2} u^{-2} \sum_{k=0}^{\infty}(k-y)^{2} \frac{y^{k}}{k !}=C(x) \delta^{-2} u^{-2} y e^{-x u+y} \\
=C(x) \delta^{-2} u^{-1} x(1+F(u) / u) e^{A / u} \exp \left(x u\left(e^{A / u}-1\right)+x F(u) e^{A / u}\right) \\
=O\left(u^{-1}\right), \text { as } u \rightarrow \infty
\end{aligned}
$$

This completes the proof of (3.3) and inasmuch as our estimates are uniformly in $x \in[a, b],(3.3)$ holds uniformly in each interval of continuity $[a, b]$ of $f(t)$.

4. Some sequence-to-function summability methods. Throughout this section we assume that $\left\{w_{i}(t)\right\}$ satisfies (1.3).

Let $\phi \in C^{\infty}(0, \infty)$ and denote $w=\left\{w_{i}(t)\right\}$. The $[J ; w ; \phi(x)]$ transform of the sequence $\left\{s_{n}\right\}(n \geqq 0)$ is defined by

$$
t(x)=\sum_{k=0}^{\infty} \psi_{k}(0 ; x) \frac{G_{k} \phi(x)}{w_{k}(x)} s_{k}, \quad x>0,
$$

provided the series on the right-hand side converges for all sufficiently large $x$. The sequence $\left\{s_{n}\right\}$ is said to be summable to $s$ by $[J ; w ; \phi(x)]$ if $\lim _{x \rightarrow \infty} t(x)=s$.

First we characterize conservative and regular $[J ; w ; \phi(x)]$ methods.

THEOREM 8. The $[J ; w ; \phi(x)]$ method is conservative if and only if $\phi(t)$ possesses the representation $(1.14)$ where $\alpha(x)$ is of bounded variation in $[0, \infty)$. It is regular if and only if in addition

$$
\alpha(0)=\alpha(0+) \text { and } \alpha(\infty)-\alpha(0)=1 .
$$

In the case $w_{i}(t) \equiv 1, i=0,1,2, \ldots$, the $[J ; w ; \phi(x)]$ method reduced to the [ $J, \phi(x)]$ method of Jakimovski [3]. Theorem 8 in this case was proved in [3]. 
Proof. It is well known that for the $[J ; w ; \phi(x)]$ method to be conservative it is necessary and sufficient that (1.12) be satisfied and that the following limits exist:

$$
\begin{gathered}
\lim _{x \rightarrow \infty} \sum_{k=0}^{\infty} \psi_{k}(0 ; x) \frac{G_{k} \phi(x)}{w_{k}(x)}, \\
\lim _{x \rightarrow \infty} \psi_{k}(0 ; x) \frac{G_{k} \phi(x)}{w_{k}(x)}, \quad k=0,1,2, \ldots
\end{gathered}
$$

Since (1.12) and (1.14) are equivalent, we only have to show that (1.14) implies the existence of the limits in (4.3) and (4.4). Now by (2.10), (2.12) and (3.6) it follows by Lebesgue's dominated convergence theorem that

$$
\begin{aligned}
\sum_{k=0}^{\infty} \psi_{k}(0 ; x) \frac{G_{k} \phi(x)}{w_{k}(x)} & =\sum_{k=0}^{\infty} \int_{0}^{\infty} \psi_{k}(0 ; x) \frac{G_{k} \psi(x ; u)}{w_{k}(x)} d \alpha(u) \\
& =\int_{0}^{\infty} \sum_{k=0}^{\infty} \psi_{k}(0 ; x) \frac{G_{k} \psi(x ; u)}{w_{k}(x)} d \alpha(u) \\
& =\alpha(\infty)-\alpha(0) .
\end{aligned}
$$

Also it follows by (3.4) and (3.5) that, for each $u>0$,

$$
\begin{aligned}
0 & \leqq \lim _{x \rightarrow \infty} \psi_{k}(0 ; x) \frac{G_{k} \psi(x ; u)}{w_{k}(x)} \\
& \leqq \lim _{x \rightarrow \infty} \frac{(x+F(\infty))^{k}}{k !} u^{k} e^{-u(x-2 F(\infty))}=0, \quad k=0,1,2, \ldots,
\end{aligned}
$$

and

$$
G_{k} \psi(x ; 0)=0, \quad k=1,2, \ldots, \quad \psi(x ; 0)=1
$$

Therefore,

$$
\lim _{x \rightarrow \infty} \psi_{k}(0 ; x) \frac{G_{k} \phi(x)}{w_{k}(x)}=0, \quad k=1,2, \ldots
$$

and

$$
\lim _{x \rightarrow \infty} \phi(x)=\alpha(0+)-\alpha(0)
$$

From (4.5) and (4.6) we see that the method is regular if and only if, in addition, (4.2) is satisfied. This completes our proof.

A totally regular method is one that sums a sequence $\left\{s_{n}\right\}(n \geqq 0)$, to $s$, whenever $s_{n} \rightarrow s$ both for $s$ finite and for $s$ infinite. We conclude this paper with a characterization of the totally regular $[J ; w ; \phi(x)]$ methods.

THEOREM 9. A regular $[J ; w ; \phi(x)]$ method is totally regular if and only if $\phi(x)$ is a GCM function in $[0, \infty)$, i.e. if and only if $\alpha(u)$ of the representation (1.14) is nondecreasing. 
Theorem 9 for the $[J, \phi(x)]$ methods was proved by Lee Lorch and the author [6].

Proof. Sufficiency of the condition is obvious. Conversely, assume that the $[J ; w ; \phi(x)]$ method is totally regular. Then by a theorem of H. Hurwitz (see [5])

$$
\lim _{x \rightarrow \infty} \sum_{k=0}^{\infty} \psi_{k}(0 ; x)\left[\frac{\left|G_{k} \phi(x)\right|}{w_{k}(x)}-\frac{G_{k} \phi(x)}{w_{k}(x)}\right]=0 .
$$

Hence, for each $0<v<\infty$,

$$
\lim _{x \rightarrow \infty} \sum_{k \leqq v x} \psi_{k}(0 ; x)\left[\frac{\left|G_{k} \phi(x)\right|}{w_{k}(x)}-\frac{G_{k} \phi(x)}{w_{k}(x)}\right]=0 .
$$

Now we may assume that $\alpha(u)$ is normalized. Then by (2.17) and the discussion at the end of the proof of Theorem 4 , if $v$ is a point of continuity of $\alpha(u)$, then

$$
\lim _{x \rightarrow \infty} \sum_{k \leqq v x} \psi_{k}(0 ; x) \frac{G_{k} \phi(x)}{w_{k}(x)}=\alpha(v)
$$

and

$$
\begin{aligned}
\int_{0}^{v}|d \alpha(u)| & \leqq \liminf _{x \rightarrow \infty} \int_{0}^{v}\left|d \alpha_{x}(u)\right| \leqq \limsup _{x \rightarrow \infty} \sum_{k \leqq v x} \psi_{k}(0 ; x) \frac{\left|G_{k} \phi(x)\right|}{w_{k}(x)} \\
& \leqq \limsup _{x \rightarrow \infty} \int_{0}^{\infty} \sum_{k \leqq v x} \psi_{k}(0 ; x) \frac{G_{k} \psi(x ; u)}{w_{k}(x)}|d \alpha(u)| .
\end{aligned}
$$

By Theorem 7, the last limit is equal to $\int_{0}^{v}|d \alpha(u)|$, hence

$$
\lim _{x \rightarrow \infty} \sum_{k \leqq v x} \psi_{k}(0 ; x) \frac{\left|G_{k} \phi(x)\right|}{w_{k}(x)}=\int_{0}^{v}|d \alpha(u)| \text {. }
$$

Combining (4.8) and (4.9), (4.7) yields

$$
\int_{0}^{v}|d \alpha(u)|-\alpha(u)=0, \quad \text { for all points of continuity } v
$$

of $\alpha(u)$, whence $\alpha(u)$ is nondecreasing.

\section{REFERENCES}

1. D. Amir and Z. Ziegler, On generalized absolutely monotone functions, Israel J. Math. 7 (1969), 137-146. MR 42 \#4681.

2. I. I. Hirschman and D. V. Widder, The convolution transform, Princeton Univ. Press, Princeton, N. J., 1955. MR 17, 479.

3. A. Jakimovski, The sequence-to-function analogues to Hausdorff transformations, Bull. Res. Council Israel Sect. F8F (1960), 135-154. MR 23 \#A3391.

4. S. Karlin and W. J. Studden, Tchebycheff systems: with application in analysis and statistics, Pure and Appl. Math., vol. 15, Interscience, New York, 1966. MR 34 \#4757.

5. S. Karlin and Z. Ziegler, Generalized absolutely monotone functions, Israel J. Math. 3 (1965), 173-180. MR 34 \#2805. 
6. D. Leviatan and L. Lorch, A characterization of totally regular $[J, f(x)]$ transforms, Proc. Amer. Math. Soc. 23 (1969), 315-319. MR 39 \#7320.

7. W. J. Studden, A generalization of Bernstein's theorem and a differential inversion formula, Trans. Amer. Math. Soc. 142 (1969), 81-92. MR 39 \#7351.

8. O. Szász, Generalization of S. Bernstein's polynomials to the infinite interval, J. Res. Nat. Bur. Standards 45 (1950), 239-245; Collected Mathematical papers, Department of Mathematics, University of Cincinnati, Cincinnati, Ohio; Hafner, New York, 1955. MR 13, 648; MR 16, 552.

Department of Mathematics, University of Illinois, Urbana, Illinois 61801

Department of Mathematics, Tel Aviv University, Tel Aviv, Israel 